\title{
Inovação na Educação Inclusiva: Estado da Arte em 2017
}

\author{
Innovation in Inclusive Education: State of Art in 2017
}

\author{
Herculano Henriques Chingui Chitungo*a; Vanderleia Benedet Reus'a Helio Aisenberg Ferenhof ${ }^{\text {b }}$ \\ Simone Meister S. Bilessimo ${ }^{\mathrm{b}}$ : Andrea Cristina Trierweiller ${ }^{\mathrm{b}}$
}

Universidade Federal de Santa Catarina, Programa de Pós-Graduação Stricto Sensu em Tecnologias de Informação e Comunicação, SC, Brasil. bUniversidade Federal de Santa Catarina, SC, Brasil.

*E-mail: herculano.henriques@gmail.com

\begin{abstract}
Resumo
O objetivo deste trabalho é revisar a produção científica sobre inovação em educação inclusiva, como forma de entender sua existência, estabelecendo o atual conhecimento relacionado ao tema que está sendo estudado na bibliografia. O estudo consiste em uma revisão sistemática de documentos acadêmicos revisados por pares, que abordam a inovação e educação inclusiva. O método de pesquisa utilizado foi o SystematicSearchFlow (SSF). Os bancos de dados utilizados foram Compendex, Scopus e Web of Science, que executam a consulta sobre o tópico, palavras-chave e resumos. A revisão resultou em 94 documentos, sendo 23 duplicados, resultando em 71 documentos, sem delimitação temporal de publicação. Após o exame do título, resumos e palavras-chave dos respectivos documentos, para se certificar de que eles corresponderam ao nosso interesse, o número de documentos foi reduzido para 58, os quais cumpriram nossos critérios e foram analisados. Este estudo, embora não tenha permitido uma cobertura completa de todos os documentos existentes revisados por pares, o seu processo de revisão abrangeu uma grande proporção de estudos disponíveis. Não somente demonstra a relevância de diferentes abordagens para contribuir com a literatura de inovação em educação inclusiva, mas também diretrizes promissoras para futuras pesquisas.
\end{abstract}

Palavras-chave: Inovação. Educação Inclusiva. Revisão Sistemática.

\begin{abstract}
The aim of this work is to review the scientific production on innovation in inclusive education, as a way to understand its existence, establishing the current knowledge related to this subject that is being currently studied and available in the literature. The study consists of a systematic review based on peer-reviewed academic papers addressing innovation and inclusive education. The search method used was SystematicSearchFlow (SSF). The databases used were Compendex, Scopus and Web of Science, which run a search on the topic, keywords and abstracts. The review search resulted in 94 documents, which 23 were duplicates, resulting in 71 documents, with no temporal delimitation of publication. After examining the title, abstracts and keywords of the respective documents, to make sure they matched our interest, the number of documents was reduced to 58 documents that met our criteria and were analyzed. Although this study did not allow full coverage of all existing peer-reviewed documents, the review process covered a large proportion of available studies. Not only does it demonstrates the relevance of different approaches to contribute to the literature of innovation in inclusive education, but also support promising guidelines for future research.
\end{abstract}

Keywords: Innovation. Inclusive Education. Systematic Review.

\section{Introdução}

A inovação tem sido considerada como uma espécie de motor para o desenvolvimento econômico e social é essencial para a competitividade (SCHUMPETER, 1997). Conforme o NMC/CoSN Horizon Report: 2017 K-12 Edition, a inovação nas escolas provocou uma tendência para os paradigmas centrados no aluno, nos quais os alunos criam habilidades de pensamento crítico em ambientes que imitam o mundo real (FREEMAN et al. 2017). Ainda segundo o relatório, a missão das escolas deve ser reinventada para refletir a mentalidade ágil e colaborativa de que os alunos precisarão para prosperar no mundo de hoje. Os líderes escolares estão usando fortes declarações de missão e visão como a "estrela orientadora" para transformar a cultura, o currículo e as operações para apoiar um modelo escolar inovador (FREEMAN et al., 2017).

Durante décadas a fio, a escola que emergiu da modernidade manteve praticamente inalteráveis os seus processos, recorrendo quase exclusivamente às tecnologias associadas à leitura e à escrita para suportá-los (FINO, 2011). Tendo em mente a gama de ferramentas pedagógicas de apoio à aprendizagem, as Tecnologias de Informação e Comunicação - TIC geram novos valores, formas de interação e comportamento social (MADARIAGA; RUBIO, 2012). Nesse contexto, nos últimos anos, temos acompanhado a integração de novas tecnologias na educação, nomeadamente TIC, com a tendência de mudar a escola, ou seja, inovar em educação.

Segundo Santos (2015), os estudos que buscam a análise da produção científica são importantes, pois apresentam a fundamentação daideia de ser imprescindível aos pesquisadores estarem atentos para a forma que o conhecimento foi gerado no seu campo de estudo. Esta pesquisa tem como objetivo revisar a produção científica sobre inovação em educação inclusiva, 
como forma de entender sua existência e estabelecer o atual conhecimento relacionado ao tema que está sendo estudado na bibliografia.

Em Sandobal et al. (2002), pode-se definir a educação inclusiva como o processo para tratar de garantir a aprendizagem e a participação de todos os alunos na vida escolar do centro, com particular atenção aos mais vulneráveis. Podemos, portanto, concluir que as crianças e jovens com deficiências e incapacidades sofrem duplamente de disfunções gerais do sistema de ensino e das dificuldades de aprendizagem relacionadas com as suas limitações próprias. A resposta a esses problemas é, naturalmente, o prosseguimento determinado do esforço para construir uma escola de qualidade. Uma escola de qualidade é, incontornavelmente, uma escola inclusiva (CAPUCHA, 2010).

Nesse contexto, faz-se necessário propor novos fluxos de trabalho e ferramentas pedagógicas para atender às demandas sugeridas pela política de inclusão na educação, para permitir e apoiar com sucesso o processo de aprendizagem às pessoas com deficiência, bem como para melhorar a qualidade de vida, independência e o seu desenvolvimento integral (ALVES et al., 2015).

O processo de mudança e inovação em que a escola embarcou depende do compromisso contínuo de certos professores (ALONSO, 2013).

O estudo da evolução da inovação e a diversidade de seus tipos possibilitam apresentar o sistema de classificação da influência da inovação, a força e a escala do impacto em todo o sistema educacional (RYAPISOVA, 2017). A política de inclusão na educação carece de estruturas metodológicas e recursos tecnológicos para auxiliar estudantes com deficiência durante seu processo de ensino-aprendizagem (ALVES et al., 2015).

Portanto, intensas atividades de pesquisa nessa área ainda são justificadas. Desse modo, dada a importância do tema desta pesquisa, a análise sistemática da área de inovação e educação inclusiva permite perceber melhores resultados no desenvolvimento de soluções de inclusão na educação. Este artigo procura responder a seguinte questão: com base nas publicações científicas sobre esse tema, quais foram os principais achados desses estudos?

\section{Desenvolvimento}

\subsection{Metodologia}

Os procedimentos metodológicos utilizados para este estudo compreendem duas etapas: Na primeira, foi realizada uma revisão sistemática da literatura, para reconhecer o estado da arte sobre o assunto. $\mathrm{Na}$ segunda, realizou-se a análise de conteúdo composta por: 1) Pré-análise; 2) Material de exploração ou codificação e 3) o tratamento dos resultados, inferência e interpretação, conforme recomendado por Bardin (2011). Nossa revisão sistemática seguiu os seis princípios de uma revisão sistemática propostos por Jesson, Matheson e
Lacey (2011), que são os seguintes: 1) Mapeamento do campo mediante uma análise de escopo; 2) Pesquisa exaustiva; 3) Avaliação de qualidade, que compreende a leitura e seleção dos trabalhos; 4) Extração de dados, que se refere à coleta e captura de dados relevantes em uma planilha pré-projetada; 5) Síntese, que compreende a síntese dos dados extraídos para mostrar o conhecido e para fornecer a base para estabelecer o desconhecido e 6) Escrever.

Opttou-se pelo método SystematicSearchFlow (SSF) para fazer a revisão de literatura, assim como analisar os resultados. Vale destacar que esse não é o único método. $\mathrm{O}$ método SSF foi desenvolvido com base na análise de vários cursos, artigos, métodos, frameworks, sistemáticas e melhores práticas que lidam com revisão de literatura e o resultado da mesma (FERENHOF; FERNANDES, 2016, p.6). O método SSF é composto por 4 fases e 8 atividades, conforme pode ser observado na Figura 1. Além disso, também foi utilizado o software EndNote X8 para gerenciar e tratar as referências coletadas. Essa ferramenta é um gerente de referência produzido pela Thomson Scientific que pode ser integrado com os bancos de dados consultados. O EndNote X8 facilita a pesquisa e a escrita científica, reunindo referências de bancos de dados online, importando metadados e agrupando-os de várias maneiras.

\section{Figura 1 - Método SSF - SystematicSearchFlow}

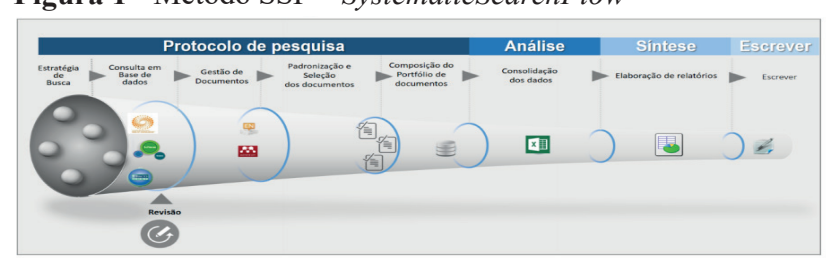

Fonte: Ferenhof e Fernandes (2016).

O primeiro passo foi o de mapear a literatura, compondo as questões de pesquisa de interesse, as palavras-chave e um conjunto de critérios de inclusão e exclusão. A consulta para essa busca foi ("inclusive education" AND innovation). Os critérios de inclusão foram documentos acadêmicos revisados por pares em inglês, português, francês e espanhol, sem delimitação temporal de publicação, e os bancos de dados utilizados foram Compendex, Scopus e Web of Science, que executam a consulta sobre o tópico, palavras-chave ou resumos. Foram representados como critérios de exclusão: a literatura cinzenta, como relatórios, livros e pesquisas não acadêmicas, e conteúdo em outros idiomas diferentes dos apresentados. Além disso, uma folha de cálculo foi produzida, consistindo em aspectos-chave relacionados ao objetivo da pesquisa.

Um dos autores acessou os três bancos de dados e procurou as pesquisas, usando a consulta resultante das combinações dos setores de palavras-chave. Buscamos combinações dessas palavras-chave no título e no resumo. Destacamos que a pesquisa nas bases de dados foi realizada em 29 de agosto de 2017, e retornaram 94 documentos, sendo 23 duplicados, 
resultando em 71 .

Cada um dos autores examinou o título, resumos e palavras-chave dos respectivos documentos, para se certificar de que corresponderam ao nosso interesse. Isso reduziu o número de documentos para 58, os quais cumpriram aos nossos critérios e foram analisados.

Os 58 artigos foram lidos pelos autores e codificados de acordo com os critérios de análise de conteúdo especificados por Bardin (2011).

Na sequência, os dados individuais foram sintetizados em uma única folha de cálculo. Mais tarde, cada autor trabalhou de forma independente na folha mesclada para verificar a consistência quanto à codificação da unidade de contexto e unidade de registro. Nossas diferentes compreensões foram compartilhadas e discutidas durante nossos períodos de discussão, os quais levaram a uma redução adicional da quantidade de documentos.

\subsection{Resultados e Discussão}

O portifólio resultante da busca sistemática será apresentado (Quadro 1) e, na sequência, os resultados serão discutidos.

Quadro 1 - Descrição dos trabalhos analisados

\begin{tabular}{|c|c|c|c|c|c|}
\hline $\mathbf{N}^{\mathbf{0}}$ & Ano & Autor & Título & Fonte da Publicação & Citações* \\
\hline 1 & 2016 & $\begin{array}{l}\text { Adayeva e } \\
\text { Satkaliyeva }\end{array}$ & $\begin{array}{l}\text { The innovative management in the field of inclusive } \\
\text { education and its impact on the public health in the } \\
\text { Republic of Kazakhstan }\end{array}$ & $\begin{array}{l}\text { International Journal of } \\
\text { Economics and Financial Issues }\end{array}$ & 0 \\
\hline 2 & 2013 & Alonso, R. F. & $\begin{array}{l}\text { Miguel Catalan secondary school: the strength of } \\
\text { normalcy }\end{array}$ & Revista de Educacion & 3 \\
\hline 3 & 2015 & Alves et al. & $\begin{array}{l}\text { Exploring technological innovation towards } \\
\text { inclusive education: building digital games - an } \\
\text { interdisciplinary challenge }\end{array}$ & $\begin{array}{l}\text { 5th International Conference } \\
\text { on New Horizons in Education } \\
\text { (INTE) }\end{array}$ & 0 \\
\hline 4 & 2016 & $\begin{array}{l}\text { Barba-Martin e } \\
\text { Barba }\end{array}$ & $\begin{array}{l}\text { An ongoing collaborative teacher training through } \\
\text { action research. A wayofchangingclassroompractices }\end{array}$ & $\begin{array}{l}\text { Contextos Educativos-Revista } \\
\text { de Educacion }\end{array}$ & 2 \\
\hline 5 & 2013 & Bardy et al. & $\begin{array}{l}\text { Objects for learning as educational resources in } \\
\text { inclusive contexts: support for distance teacher } \\
\text { education }\end{array}$ & $\begin{array}{l}\text { Revista Brasileira de Educação } \\
\text { Especial }\end{array}$ & 0 \\
\hline 6 & 2014 & Bari et al. & $\begin{array}{l}\text { The readiness of special education trainees to teach } \\
\text { in inclusive program }\end{array}$ & InternationalEducationStudies & 0 \\
\hline 7 & 2013 & Batanero, J. M. F. & Teaching competences and inclusive education & \begin{tabular}{|l|} 
Revista Electronica de \\
Investigacion Educativa \\
\end{tabular} & 43 \\
\hline 8 & 2015 & Benigno et al. & $\begin{array}{l}\text { A web-based knowledge hub for special and } \\
\text { inclusive education }\end{array}$ & $\begin{array}{l}\text { International Journal of } \\
\text { Emerging Technologies in } \\
\text { Learning }\end{array}$ & 0 \\
\hline 9 & 2016 & Bernal e Arteaga & $\begin{array}{l}\text { The inquiry of pedagogy in the context of the } \\
\text { inclusive education sociocritical movements }\end{array}$ & Prisma Social & 0 \\
\hline 10 & 2014 & Blasco et al. & ICT as a model of sensory training in physiotherapy & $\begin{array}{l}\text { 8th International Technology, } \\
\text { Education and Development } \\
\text { Conference (INTED) } \\
\end{array}$ & 0 \\
\hline 11 & 2016 & Bosse, I. K. & $\begin{array}{l}\text { Cloud computing in european schools - the impact } \\
\text { on inclusive education }\end{array}$ & $\begin{array}{l}\text { 15th International Conference } \\
\text { on Computers Helping People } \\
\text { with Special Needs (ICCHP) } \\
\end{array}$ & 0 \\
\hline 12 & 2010 & Capucha, L. & $\begin{array}{l}\text { Innovation and social justice: active policies for } \\
\text { inclusion in education }\end{array}$ & $\begin{array}{l}\text { Sociologia, Problemas e } \\
\text { Práticas }\end{array}$ & 24 \\
\hline 13 & 2012 & Cortes, R. J. & $\begin{array}{l}\text { Inclusive education and initial teacher training: } \\
\text { evaluation of a didactic innovation based on } \\
\text { filmmaking from a student's perspective }\end{array}$ & Revista de Educacion & 6 \\
\hline 14 & 2014 & Deluca et al. & $\begin{array}{l}\text { Promoting the provision of inclusive primary } \\
\text { education for children with disabilities in } \\
\text { mashonaland west province, Zimbabwe }\end{array}$ & $\begin{array}{l}\text { 8th International Technology, } \\
\text { Education and Development } \\
\text { Conference (INTED) }\end{array}$ & 1 \\
\hline 15 & 2015 & Djukic et al.. & $\begin{array}{l}\text { Inclusive education system in Serbia between theory } \\
\text { and practice }\end{array}$ & $\begin{array}{l}\text { 7th International Conference on } \\
\text { Education and New Learning } \\
\text { Technologies (EDULEARN) } \\
\end{array}$ & 0 \\
\hline 16 & 2009 & Echeita et al. & $\begin{array}{l}\text { Paradoxes and dilemmas in the inclusive education } \\
\text { process in Spain }\end{array}$ & Revista de Educacion & 5 \\
\hline 17 & 2011 & $\begin{array}{l}\text { Fernandez, M. } \\
\text { S. J. }\end{array}$ & $\begin{array}{l}\text { The innovative model of diversity and high } \\
\text { capabilities attention. "agora model" }\end{array}$ & $\begin{array}{l}\text { 3rd International Conference on } \\
\text { Education and New Learning } \\
\text { Technologies (EDULEARN) }\end{array}$ & 0 \\
\hline 18 & 2014 & $\begin{array}{l}\text { Fernandez- } \\
\text { Batanero, J. M. }\end{array}$ & $\begin{array}{l}\text { Strategies for inclusion in the face of social } \\
\text { exclusion. Case study in Andalusia (Spain) }\end{array}$ & $\begin{array}{l}\text { European Journal of Special } \\
\text { Needs Education }\end{array}$ & 4 \\
\hline
\end{tabular}




\begin{tabular}{|c|c|c|c|c|c|}
\hline 19 & 2015 & $\begin{array}{l}\text { Fernandez- } \\
\text { Batanero e Ruiz }\end{array}$ & $\begin{array}{l}\text { Teacher attitudes towards ICT in the context of } \\
\text { inclusive education }\end{array}$ & \begin{tabular}{|l|} 
3rd International Conference on \\
Technological Ecosystems for \\
Enhancing Multiculturality \\
\end{tabular} & 0 \\
\hline 20 & 2000 & Fisher et al. & $\begin{array}{l}\text { The resilience of changes promoting inclusiveness in } \\
\text { an urban elementary school }\end{array}$ & ElementarySchoolJournal & 49 \\
\hline 21 & 2011 & $\begin{array}{l}\text { Florian e } \\
\text { Becirevic }\end{array}$ & $\begin{array}{l}\text { Challenges for teachers' professional learning for } \\
\text { inclusive education in central and eastern Europe and } \\
\text { the commonwealth of independent states }\end{array}$ & Prospects & 15 \\
\hline 22 & 2015 & Gavira e Morina & $\begin{array}{l}\text { Hidden voices in higher education: inclusive policies } \\
\text { and practices in social science and law classrooms }\end{array}$ & $\begin{array}{l}\text { International Journal of } \\
\text { Inclusive Education }\end{array}$ & 12 \\
\hline 23 & 2004 & Ghesquière et al. & $\begin{array}{l}\text { The usefulness of qualitative case studies in research } \\
\text { on special needs education }\end{array}$ & $\begin{array}{l}\text { International Journal of } \\
\text { Disability, Development and } \\
\text { Education }\end{array}$ & 70 \\
\hline 24 & 2016 & $\begin{array}{l}\text { González Cortés } \\
\text { et al. }\end{array}$ & $\begin{array}{l}\text { Invisible barriers? College student's attitudes } \\
\text { towards peers with disabilities }\end{array}$ & $\begin{array}{l}\text { Revista Complutense de } \\
\text { Educacion }\end{array}$ & 0 \\
\hline 25 & 2014 & Hostins e Jordão & $\begin{array}{l}\text { School inclusion policy and curricular practices: } \\
\text { teaching strategies for target audience conceptual } \\
\text { elaboration of special education }\end{array}$ & $\begin{array}{l}\text { Education Policy Analysis } \\
\text { Archives }\end{array}$ & 0 \\
\hline 26 & 2016 & Infantes et al. & Inclusion as an element of empowerment & Prisma Social & 0 \\
\hline 27 & 2015 & Kavkler et al. & $\begin{array}{l}\text { Inclusive education for children with specific } \\
\text { learning difficulties: Analysis of opportunities and } \\
\text { barriers in inclusive education in Slovenia }\end{array}$ & $\begin{array}{l}\text { Center for Educacional Policy } \\
\text { Studies Journal }\end{array}$ & 5 \\
\hline 28 & 2010 & $\begin{array}{l}\text { Krasnomovets, } \\
\text { V. A. }\end{array}$ & $\begin{array}{l}\text { Introducing innovations in education as a } \\
\text { precondition for social development of disabled } \\
\text { people in Ukraine }\end{array}$ & Actual Problems of Economics & 0 \\
\hline 29 & 2017 & Liu et al. & $\begin{array}{l}\text { Research on the computer digital education of } \\
\text { special children based on the theory of multiple } \\
\text { intelligences }\end{array}$ & Agro Food Industry Hi-Tech & 0 \\
\hline 30 & 2016 & Magnusson, G. & $\begin{array}{l}\text { Representations of special support. Independent } \\
\text { schools' descriptions of special educational provision }\end{array}$ & $\begin{array}{l}\text { European Journal of Special } \\
\text { Needs Education }\end{array}$ & 2 \\
\hline 31 & 2012 & Marin, S. M. & $\begin{array}{l}\text { Change and innovation in the educational policies } \\
\text { and strategies for human resources development. }\end{array}$ & \begin{tabular}{|l|} 
Cyprus International \\
Conference on Educational \\
Research \\
\end{tabular} & 4 \\
\hline 32 & 2015 & Mesa e Garcia & $\begin{array}{l}\text { Adaptation of Index for Inclusion to the field of } \\
\text { higher education: Preliminary study }\end{array}$ & Intangible Capital & 1 \\
\hline 33 & 2011 & Moliner et al. & $\begin{array}{l}\text { Inclusive cultures, policies and practices in Spanish } \\
\text { compulsory secondary education schools: teachers' } \\
\text { perceptions in ordinary and specific teaching } \\
\text { contexts }\end{array}$ & $\begin{array}{l}\text { International Journal of } \\
\text { Inclusive Education }\end{array}$ & 21 \\
\hline 34 & 2017 & \begin{tabular}{|l|} 
Montero e \\
Jormanainen
\end{tabular} & $\begin{array}{l}\text { Theater meets robot - toward inclusive STEAM } \\
\text { education }\end{array}$ & $\begin{array}{l}\text { International Conference on } \\
\text { Educational Robotics }\end{array}$ & 0 \\
\hline 35 & 2006 & Mundia, L. & $\begin{array}{l}\text { Aggressive behaviour among Swazi upper primary } \\
\text { and junior secondary students: Implications for } \\
\text { ongoing educational reforms concerning inclusive } \\
\text { education }\end{array}$ & $\begin{array}{l}\text { International Journal of Special } \\
\text { Education }\end{array}$ & 13 \\
\hline 36 & 2006 & Nicolaidou et al. & $\begin{array}{l}\text { Promoting inclusive practices in primary schools } \\
\text { in Cyprus: Empowering pupils to build supportive } \\
\text { networks }\end{array}$ & $\begin{array}{l}\text { European Journal of Special } \\
\text { Needs Education }\end{array}$ & 15 \\
\hline 37 & 2009 & Ntombela, S. & $\begin{array}{l}\text { Are we there yet? Towards the development of } \\
\text { inclusive education in one district in Kwazulu-Natal, } \\
\text { South Africa }\end{array}$ & $\begin{array}{l}\text { International Journal of } \\
\text { Learning }\end{array}$ & 4 \\
\hline 38 & 2016 & Olmos, E. F. T. & $\begin{array}{l}\text { Designing enriched learning environments: a cross- } \\
\text { disciplinary approach to social innovation }\end{array}$ & $\begin{array}{l}\text { Design Education: } \\
\text { Collaboration and Cross- } \\
\text { Disciplinary }\end{array}$ & 0 \\
\hline 39 & 2014 & $\begin{array}{l}\text { Omolade e } \\
\text { Solomon }\end{array}$ & $\begin{array}{l}\text { Perception and use of computer and mobile phones } \\
\text { technologies among visually impaired residents of } \\
\text { Lagos state, Nigeria }\end{array}$ & $\begin{array}{l}\text { Edulearn 14: 6th International } \\
\text { Conference on Education and } \\
\text { New Learning Technologies } \\
\end{array}$ & 0 \\
\hline 40 & 1999 & Parrilla, A. & $\begin{array}{l}\text { Educational innovations as a school answer to } \\
\text { diversity }\end{array}$ & $\begin{array}{l}\text { International Journal of } \\
\text { Inclusive Education }\end{array}$ & 23 \\
\hline 41 & 2017 & Parrilla et al. & $\begin{array}{l}\text { Critically reviewing how we do research into } \\
\text { inclusive education: four projects with an educational } \\
\text { and social approach }\end{array}$ & $\begin{array}{l}\text { International Journal of } \\
\text { Inclusive Education }\end{array}$ & 0 \\
\hline
\end{tabular}




\begin{tabular}{|c|c|c|c|c|c|}
\hline 42 & 2012 & Pernía et al. & $\begin{array}{l}\text { Student voice in school improvement: Children as } \\
\text { researchers in primary school }\end{array}$ & Revista de Educacion & 13 \\
\hline 43 & 2016 & Pernía et al. & $\begin{array}{l}\text { Growing student voice in curriculum decisions at the } \\
\text { university }\end{array}$ & $\begin{array}{l}\text { Journal of Research in Special } \\
\text { Educational Needs }\end{array}$ & 15 \\
\hline 44 & 2014 & Popescu et al. & $\begin{array}{l}\text { Eyeschool: an educational assistive technology for } \\
\text { people with disabilities - passing from single actors } \\
\text { to multiple-actor environment }\end{array}$ & $\begin{array}{l}\text { Computers Helping People } \\
\text { with Special Needs, Icchp } \\
\text { 2014, Pt Ii }\end{array}$ & 4 \\
\hline 45 & 2009 & Rafal, J. & $\begin{array}{l}\text { Inclusive education: Innovation or merely the } \\
\text { reproduction of hegemonic ideology? }\end{array}$ & \begin{tabular}{|l|} 
International Journal of \\
Learning
\end{tabular} & 4 \\
\hline 46 & 2000 & Rainforth, B. & $\begin{array}{l}\text { Preparing teachers to educate students with severe } \\
\text { disabilities in inclusive settings despite contextual } \\
\text { constraints }\end{array}$ & $\begin{array}{l}\text { Journal of the Association for } \\
\text { Persons with Severe Handicaps }\end{array}$ & 45 \\
\hline 47 & 2010 & Reicher, $\mathrm{H}$. & $\begin{array}{l}\text { Building inclusive education on social and emotional } \\
\text { learning: challenges and perspectives - a review }\end{array}$ & $\begin{array}{l}\text { International Journal of } \\
\text { Inclusive Education }\end{array}$ & 31 \\
\hline 48 & 2014 & Reupert et. al. & $\begin{array}{l}\text { Enablers for inclusion: the perspectives of parents of } \\
\text { children with autism spectrum disorder }\end{array}$ & $\begin{array}{l}\text { Australasian Journal of Special } \\
\text { Education }\end{array}$ & 6 \\
\hline 49 & 2014 & $\begin{array}{l}\text { Rosin-Pinola e } \\
\text { Del Prette. } \\
\end{array}$ & $\begin{array}{l}\text { School inclusion, teacher training and consultation } \\
\text { based on educational social skills }\end{array}$ & $\begin{array}{l}\text { Revista Brasileira de Educação } \\
\text { Especial }\end{array}$ & 0 \\
\hline 50 & 2017 & Ryapisova, A. G. & Inclusive education as a systemic innovation & $\begin{array}{l}\text { Novosibirsk State Pedagogical } \\
\text { University Bulletin } \\
\end{array}$ & 1 \\
\hline 51 & 2015 & Sanchez et al. & $\begin{array}{l}\text { Evaluation in primary education as starting point in } \\
\text { the development of inclusive improvement plan in } \\
\text { the Region of Murcia }\end{array}$ & \begin{tabular}{|l|} 
Revista Electronica \\
Interuniversitaria de Formacion \\
del Profesorado
\end{tabular} & 14 \\
\hline 52 & 2013 & $\begin{array}{l}\text { Sánchez-Teruel e } \\
\text { Robles-Bello }\end{array}$ & $\begin{array}{l}\text { Inclusion as key to education for all: a theoretical } \\
\text { review }\end{array}$ & $\begin{array}{l}\text { Revista Espanola de } \\
\text { Orientacion y Psicopedagogia } \\
\end{array}$ & \\
\hline 53 & 2015 & $\begin{array}{l}\text { Schmidt e } \\
\text { Brown. }\end{array}$ & $\begin{array}{l}\text { Education of children with intellectual disabilities in } \\
\text { Slovenia }\end{array}$ & $\begin{array}{l}\text { Journal of Policy and Practice } \\
\text { in Intellectual Disabilities }\end{array}$ & 6 \\
\hline 54 & 2015 & Sokal e Katz & $\begin{array}{l}\text { Oh, Canada: bridges and barriers to inclusion in } \\
\text { Canadian schools }\end{array}$ & Support for Learning & 7 \\
\hline 55 & 2010 & Starcic, A. I. & Educational technology for the inclusive classroom & $\begin{array}{l}\text { Turkish Online Journal of } \\
\text { Educational Technology }\end{array}$ & 32 \\
\hline 56 & 2015 & Stoica et al. & Learn the stories from images and beyond & $\begin{array}{l}\text { Edulearn15: } 7 \text { th International } \\
\text { Conference on Education and } \\
\text { New Learning Technologies } \\
\end{array}$ & \\
\hline 57 & 2014 & Thomson, P. & $\begin{array}{l}\text { 'Scaling up' educational change: some musings on } \\
\text { misrecognition and doxic challenges }\end{array}$ & CriticalStudies in Education & 16 \\
\hline 58 & 2003 & Vislie, L. & $\begin{array}{l}\text { From integration to inclusion: focusing global trends } \\
\text { and changes in the western European societies }\end{array}$ & $\begin{array}{l}\text { European Journal of Special } \\
\text { Needs Education }\end{array}$ & 321 \\
\hline
\end{tabular}

Fonte: Dados da Pesquisa, número de citações, com base no Google Académico*

O Quadro 2 apresenta a quantidade de artigos encontrados nos bancos de dados que resumiram as estratégias pesquisadas.

Quadro 2 - Quantidadede artigos encontrados por banco de dados

\begin{tabular}{|c|c|c|}
\hline Banco de Dados & Periódicos & Documentos \\
\hline Compendex & 4 & 7 \\
\hline Scopus & 36 & 47 \\
\hline Web of Science & 29 & 40 \\
\hline
\end{tabular}

Fonte: Dados da Pesquisa.

Como forma de acompanhar os estudos ao longo do tempo, o Quadro 3 mostra a quantidade de artigos publicados por ano.

Quadro 3 - Quantidade de artigos publicados por ano

\begin{tabular}{|c|c|}
\hline Ano de Publicação & Frequência \\
\hline 1999 & 1 \\
\hline 2000 & 2 \\
\hline 2003 & 1 \\
\hline 2004 & 1 \\
\hline 2006 & 2 \\
\hline 2009 & 3 \\
\hline 2010 & 4 \\
\hline
\end{tabular}

\begin{tabular}{|c|c|}
\hline 2011 & 3 \\
\hline 2012 & 3 \\
\hline 2013 & 4 \\
\hline 2014 & 10 \\
\hline 2015 & 11 \\
\hline 2016 & 9 \\
\hline 2017 & 4 \\
\hline
\end{tabular}

Fonte: Dados da Pesquisa.

Pode-se verificar que a maioria dos documentos identificados foi publicada em 2015. Em seguida, temos como segunda maior publicação em 2014, em terceiro, 2016. O restante dos documentos possui publicação em diversos anos, variando de 1 a 4 . Ainda, sobre os aportes dos trabalhos analisados sobre inovação inclusiva na educação, são mostrados os resultados de cada estudo.

Vislie (2003) aborda a terminologia inclusão, e a diferencia de integração, referendada "na Declaração de Salamanca". O novo termo ocasionou o surgimento de uma nova agenda de políticas educacionais". Relata as ações da UNESCO (Organização das Nações Unidas para a Educação, 
a Ciência e a Cultura), na década de 90, e suas implicações nas políticas inclusivas e faz referência a algumas inovações recentes no Reino Unido. Propõe uma análise em 14 países europeus (obtidos em 1990 e 1996). Rainforth (2000) relata a necessidade de mudanças nos "distritos escolares", em como a defesa "para estudantes com deficiências severas com base no mandato ambiental menos restritivo das alterações da Lei de Educação de Pessoas com Deficiências de 1997'. Professores de professores em faculdades e universidades também devem assumir essa responsabilidade. Para Thomson (2014), "os formuladores de políticas educacionais em todo o mundo estão fortemente comprometidos com a noção de "ampliar".

Em todo o mundo, a educação para necessidades especiais está se transformando em educação inclusiva. O sucesso dessa inovação nas escolas regulares é determinado por muitos fatores (GHESQUIÈRE et al., 200). Capucha (2010) trata da inovação e justiça social através de políticas ativas para a educação inclusiva. Segundo o autor, é preciso inovação para diferenciar "escola inclusiva" de "escola miscelânea", de modo a qualificar a instituição escolar, e também a implementar políticas específicas de educação especial. Uma escola inclusiva implica mudanças em três âmbitos: o organizativo, a da intervenção didática, o de comunidade social (CORTES, 2012).

Ntombela (2009) salienta que o interesse das escolas e a postura do professor são essenciais para promoção do contexto inovador. Não basta apenas treinamento para os profissionais da educação para modificar a cultura e influenciar os distritos. Moliner et al. (2011) apresentam os indicadores relacionados à educação inclusiva, salientando "o papel dos processos de inovação e transformação nas escolas secundárias é fornecemos fatores-chave para a construção de uma escola intercultural inclusiva". Sánchez-Teruel e Robles-Bello (2013) propõem auxiliar a todos os espanhóis "profissionais e educadores (autoridades competentes, pais, professores, etc.) a ampliar a percepção do que significa educar e diversificar na sociedade atual.

Os trabalhos desenvolvidos por autores como Batanero (2013) e Bari et al. (2014) explicam a necessidade da preparação do professor para o desenvolvimento de boas práticas educacionais em relação à inclusão educacional dos alunos. Bari et al. (2014) ressaltam dizendo que a inovação no ensino e na formação prática ainda precisa ser fornecida de forma suficiente, para que os professores possam estar preparados e compreender os programas inclusivos, pois, o papel dos professores é essencial para garantir que os alunos com deficiências ganhem a oportunidade de experimentar mudanças positivas na educação. No estudo de Fisher et al. (2000), os professores de uma escola criaram um ambiente inclusivo para todos os alunos, mantendo essa inovação no meio de uma mudança administrativa. Os resultados indicaram que "a educação inclusiva foi sustentada quando os professores compartilharam a visão, os recursos estavam disponíveis, o treinamento foi fornecido e uma cultura de inclusão foi criada dentro da escola".

Florian e Becirevic (2011) identificam alguns desafios que enfrentam os interessados na preparação de professores para educação inclusiva e fornecem exemplos de projetos atuais na Europa Central e Oriental e na Comunidade de Estados Independentes, que abordam os complexos problemas associados ao fornecimento de educação inclusiva para crianças com deficiência. Além disso, a mudança do papel dos defectologistas na política e prática de inclusão também é discutida.

Marin (2012) também enfatiza o processo educacional e as formações para ampliar o conhecimento e o uso das novas tecnologias (e-Learning) e a prioridade da política do Conselho da UE (União Europeia) de forma ampla e contextualizada.

Echeita et al. (2009) mostram os resultados sobre o processo de educação inclusiva na Espanha, onde "permitem a discussão sobre alguns dos paradoxos e dilemas que os agentes desse processo enfrentam e, em qualquer caso, mostra as diferentes barreiras existentes que representam um obstáculo para aproveitar o direito a uma educação inclusiva". Djukic et al. (2015) mostram os resultados sobre a educação inclusiva sobre a teoria pedagógica e a prática no sistema educacional da Sérvia. Segundo o autor, através da aplicação de inovações pedagógicas e mudanças na política educacional é possível fornecer a implementação do direito à educação e acesso à educação para todos, sem qualquer discriminação. Adayeva e Satkaliyeva (2016) abordam o problema e as condições da educação inclusiva, através da introdução de um projeto inovador de inclusão no sistema educacional do Cazaquistão, pois segundo os autores, "a relevância e importância dos problemas de gestão e desenvolvimento das inovações neste sistema $[\ldots]$ "..

Conforme Schmidt e Brown (2015), na Eslovênia, o código da adoção da Colocação de Crianças com SpecialNeedsAct $(2000,2007)$ representa uma inovação, pois as crianças ainda estudam em ambientes separados da escola regular. Para Parrilla (1999), as escolas primárias e secundárias sevilhanas estão inovando para responder às questões da diversidade por meio de projetos. Segundo o autor, a primeira fase da pesquisa foi a identificação de todas as inovações ligadas à diversidade, realizada na província de Sevilha, durante o ano letivo 199697”, para verificar "a tipologia das inovações" e criar “ estratégias para promover a educação inclusiva nos estudos de caso da província de Sevilha”. Sanchez et al. (2015) elencam o desafio de oferecer educação de qualidade e inclusiva, por meio do processo de autoavaliação, projeto realizado em "quatro escolas primárias da Região de Múrcia (Espanha)". Utilizaram (ACADI), na qual participantes de cada uma das escolas refletiram sobre sua própria realidade e prática, revelando um diagnóstico real com pontos fracos e fortes. Segundo Nicolaidou et al. (2006), estruturas e procedimentos para os alunos se sentiram emponderados e atuaram como pesquisadores, foi uma inovação em Chipre, na resolução de problemas logísticos. 
Popescu et al. (2014) elencam o incentivo, por meio de políticas públicas da França, para a inclusão de jovens deficientes no sistema escolar e aplicação direta através da inovação técnica, com tecnologia assistiva e o auxílio nas atividades de aprendizado. Pernia et al. (2016) relatam o desenvolvimento das Escolas de Projetos de $\mathrm{P}+\mathrm{D}$ (Pesquisa e Desenvolvimento) que estão se movendo em direção à educação inclusiva na Universidade de Cantabria (Espanha)", por meio colaborativo "no desenvolvimento do currículo (Seale)", promovendo uma conexão real com o conhecimento.

Ryapisova (2017) identifica os sinais de inovação sistêmica no exemplo da introdução de práticas inclusivas nas modernas condições da modernização da educação russa. "A conclusão justifica-se de que a introdução da educação inclusiva na prática educacional geral como um sistema de inovação fundamental, estrutural, radical e multidimensional tem recursos suficientes para melhorar a qualidade e aumentar a eficiência da educação".

Rafal (2009) discute e justapõe três tipos de modelos escolares nos EUA que tentam ativamente reformar seus programas para estudantes com necessidades especiais, tanto por meio de políticas como de práticas dentro de um sistema educacional que está evoluindo e enfatiza "a falta de pesquisa diretamente, incluindo as vozes dos alunos que são mais marginalizados dentro de um ambiente de educação, é explorada para estabelecer a importância e a relevância de incluir os múltiplos pontos de vista de muitos estudantes".

Reupert et al. (2014) apresentam a participação dos pais australianos, seus pontos de vistas, das crianças com diagnóstico de transtorno do espectro autista e suas experiências de um programa de apoio em escolas inclusivas. "Os pais identificaram várias inovações, incluindo a provisão de espaço seguro, escola estruturada e tempo livre, flexibilidade em torno do cronograma, currículo e pessoal e a oferta de atividades socialmente atraentes".

Sokal e Katz (2015) relatam a inclusão em escolas canadenses, tanto acadêmicas como sociais, exploradas através das estruturas legislativas com programas inovadores para a inclusão. Magnusson (2016) diz que um argumento importante para a introdução da escolha da escola na Suécia, foi que as escolas independentes seriam uma fonte de inovação.

No Brasil, Hostins e Jordão (2014) dizem que "as políticas de inclusão escolar e as práticas curriculares direcionadas a escolarização de alunos público alvo da educação especial têm sido objeto de indagação e debate". Para Rosin-Pinola e Del Prette (2014), é necessária a reorganização das escolas brasileiras, para incluir as crianças com necessidades educacionais especiais NEE (Núcleo de Educação Especial) no processo de educação geral, apresentam o contexto histórico e legislação, inovações dos órgãos governamentais, bem como a necessidade de formação profissional.O estudo de Deluca et al. (2014) examina os resultados de uma pesquisa sobre deficiência e educação inclusiva nas escolas do Zimbabwe, na África, que examina conhecimento, atitudes e práticas de professores e famílias, bem como barreiras e desafios. Entre as principais barreiras percebidas, que impedem as crianças com deficiência de ir à escola pelos entrevistados, estão a falta de dispositivos de assistência, além da distância à escola e a falta de transporte.

Gavira e Morina (2015) centram-se especificamente nas barreiras e no apoio identificados pelos alunos com deficiência em uma universidade espanhola. Segundo os autores, "tomando como referência o modelo social da deficiência, concluímos que, para ser inclusivo, a universidade precisa comprometer-se a adotar medidas proativas que eliminem as barreiras que não permitem a aprendizagem e a participação plena dos alunos em questão". Mesa e Garcia (2015) analisam, por meio do desenho descritivo, "a adaptação do índice de inclusão ao contexto universitário na Espanha, e salientam que a universidade "ainda" é detectada como organização inflexível e necessita projeto de pesquisa colaborativo, para assim poder modificar o contexto social".

Krasnomovets (2010) considerou inovadora a formação, por meio de educação à distância, e as oportunidades que as tecnologias proporcionam para as pessoas com deficiência. Starcic (2010) introduziu o ambiente SEVERI e-learning nas escolas eslovenas. "Ao se concentrar mais especificamente no uso das TIC para alunos com necessidades especiais, o objetivo é colocar em prática os princípios de igualdade, diversidade e educação inclusiva".

Para Bardy et al. (2013), recursos como a Tecnologia Assistiva, as TIC, as Salas de Recursos Multifuncionais são fundamentais para promover a inclusão de estudantes com deficiência no ensino regular, bem como potencializar a aprendizagem de todos os estudantes.

Blasco et al. (2014) mostram uma proposta educacional inovadora usando as TIC, para alunos com limitações e dificuldades sensoriais. Alonso (2013) analisa uma experiência educacional inovadora em uma escola do ensino médio na Espanha, onde a solução foi fundamentada no envolvimento ativo de estudantes e funcionários, através da resolução de conflitos democráticos, levando inovações, como ter dois professores na mesma sala de aula, grupos interativos e uso intensivo de TIC.

Omolade e Solomon (2014) propõem a alfabetização com a informática e "o uso de tecnologias informáticas e de telefonia móvel entre deficientes visuais em comunicação efetiva, educação especial e participação em atividades sociais e on-line" na instituição superior de aprendizagem na Nigéria. A teoria da difusão da inovação, a teoria das lacunas do conhecimento e a teoria dos participantes democratas são usadas como estrutura teórica para o estudo. Utilizando o projeto de levantamento descritivo, pessoas com deficiência visual do Centro de Treinamento Profissional para cegos (VTC), Oshodi, Lagos e Anglo-WelfareAssociation for Blind (ANWAB), Yaba, Lagos foram entrevistados através de questionário auto administrado, complementado com Key 


\section{Informant Interview.}

Fernandez-Batanero e Ruiz (2015) apresentam os resultados de um estudo cujo objetivo era determinar como um professor usará e integrará as TIC em salas de aula inclusivas. O resultado mostra que os professores em geral têm uma atitude positiva em relação às TIC. Isso também promoverá políticas inclusivas e culturais entre redes de escolas e é apresentado como um fator importante no desenvolvimento de boas práticas educacionais com o apoio das TIC (FERNANDEZ-BATANERO; RUIZ, 2015).

Alves et al. (2015), usando uma abordagem interdisciplinar, exploram a inovação tecnológica para a educação inclusiva através do desenvolvimento educacional de jogos digitais acessíveis com design de interface natural e universal. Segundo os autores, "a política de inclusão na educação carece de estruturas metodológicas e recursos tecnológicos para auxiliar estudantes com deficiência durante seu processo de ensino-aprendizagem". Benigno et al. (2015) apresentam os resultados de uma pesquisa voltada para a definição de um ambiente baseado na Web para a educação especial e inclusiva. Trata-se de um repositório semântico de recursos digitais que permite armazenar e recuperar informações personalizadas e recursos digitais que cobrem suas expressões e necessidades latentes de informação.

Bosse (2016) diz que a "computação em nuvem pode ser um dos principais impulsionadores da inovação e transformação na aprendizagem e no ensino". O autor apresenta resultados de "boas práticas existentes, explorando o impacto da Cloud sobre a aprendizagem personalizada para alunos com necessidades especiais". Também "discute como os professores podem usar as novas tecnologias como componente de valor agregado na educação inclusiva".

Para Liu et al. (2017), o conceito de "educação inclusiva" de desenvolvimento generalizado e rápido, aumentaram gradualmente a preocupação social com os grupos desfavorecidos. Com o rápido desenvolvimento da tecnologia de computadores e redes, o ensino digital por computador também se tornou um importante e promissor novo campo na educação para crianças com deficiências.

Conforme Montero e Jormanainen (2017), a ciência, a tecnologia, a engenharia e a educação matemática foram expandidas pela inclusão das artes como uma área integral. Como tal, a educação STEAM é considerada como sendo inclusiva em gênero e acessível para todos os níveis de habilidades técnicas. Neste estudo, os autores tratam sobre a inclusão das artes na educação robótica através do conceito de robótica teatral. As lições aprendidas com nossos empreendimentos de robótica de teatro serão alavancadas no projeto de pesquisa e inovação Horizon 2020 e CraftLearn, onde esperamos conseguir uma integração harmoniosa de assuntos e habilidades técnicas e humanas, abrindo o caminho para uma educação motivadora e inclusiva para todos os alunos em diferentes contextos de STEAM.

Stoica et al. (2015) sugerem dizendo que "para os alunos com deficiências visuais, devem ser fornecidos materiais especiais (os mais comuns sendo os livros em Braille) e serviços, bem como tecnologia especializada que comprovam a igualdade de acesso aos currículos e informações disponíveis para os outros alunos". Das soluções escolhidas no Museu Nacional de Arte da Romênia para o Portão de pintura de Paul Signac (Saint-Tropez) e para a pintura de Guercino, São Bento e São Francisco, o autor considera dois aplicativos desenvolvidos para o Android para pessoas com deficiência visual.

Fernandez (2011) apresenta um modelo inovador de ensino colaborativo que permite a detecção e atenção de estudantes com habilidades elevadas em qualquer escola comum. Além disso, esse modelo também é dirigido a estudantes de desenvolvimento tardio e estudantes com necessidades educacionais especiais que cobrem uma ampla gama de distúrbios, de distúrbios menores a estudantes gravemente afetados.

González Cortés et al. (2016) analisam os conhecimentos, as opiniões e as atitudes dos estudantes universitários sobre os colegas com deficiência, bem como as perspectivas de estudantes com deficiência, necessárias para medir o grau de aceitação dentro das universidades.

Para Barba-Martin e Barba (2016), é necessária a parceria entre escolas e universidades através de projetos de inovação sobre a educação inclusiva. O estudo de Bernal e Arteaga (2016) trata da reflexão da Pedagogia no contexto da educação inclusiva. Segundo os autores, os estudos sobre educação inclusiva "aumentam cada vez mais os resultados que influenciam globalmente a transformação do conceito de educação com base na atenção à diversidade, como fator de inovação social". Pernía et al. (2012) fazem menção ao relatório parcial da Universidade de Cantabria em "diferentes escolas cantábricas, nas quais a voz do aluno e a educação inclusiva estão sendo usadas como base para impulsionar a melhoria da escola". Esses projetos incentivam "estudantes a ser pesquisadores", e para isso utiliza-se a abordagem etnográfica das escolas, inovando-a.

Infantes et al. (2016) apresentam um projeto de inovação pedagógica e uma experiência de serviço-aprendizagem desenvolvida ao longo de sete anos entre uma escola maternal e primária e um centro ocupacional de pessoas com diversidade funcional da província de Alicante, na Espanha. Assim, mostra um "exemplo prático de educação inclusiva no campo da diversidade onde tem sido muito presente a perspectiva de gênero".

Para Fernandez-Batanero (2014), a participação de todos [...] "é essencial para que a escola se considere como uma unidade de troca ou um espaço de pesquisa que incorpore a diferenciação em seus processos de inovação".

Kavkler et al. (2015) analisam as barreiras e oportunidades na área de SpLD (Specific Learning Difficulty) com o modelo de Ball (1994), enfatizam a universalização da educação inclusiva "proporcionando o direito legislativo e com isso, 
discutiram as garantias da assistência e dos programas de apoio às crianças com aprendizagem específicas.

Mundia (2006) inovou propondo nas escolas Swazi "educação inclusiva como parte da educação para todos e assim, beneficiar os alunos com distúrbios emocionais e comportamentais. Os cursos de preservação e inservice, a nomeação de conselheiros escolares e novas pesquisas foram elencadas como importantes para o contexto inovador. Olmos (2016) investigou as barreiras na disciplina de design industrial e desenvolvimento de produtos em uma instalação interdisciplinar focada em crianças com Síndrome de Down, Transtorno de Déficit de Atenção com Hiperatividade e Autismo Transtorno do Espectro". Aponta a necessidade de "design de produtos como ferramentas de aprendizado para gerenciar a linguagem, que significa geração e tecnologia". Ele representa uma reflexão contemporânea sobre as ideias centrais do trabalho de Victor Papanek Design para o mundo real.

Reicher (2010) abordou “questões conceituais e empíricas relacionadas aos vínculos entre aprendizagem social e emocional e educação inclusiva" com o foco centrado na pessoa, promovendo a prevenção "em configurações inclusivas da escola através de modificações de insumos e conteúdos, estratégias e processos de ensino, bem como a melhoria dos ambientes de aprendizagem". Parrilla (2017) reflete sobre "as bases conceituais e epistemológicas que compartilham quatro ações em andamento", a promoção de "processos e projetos de pesquisa e aperfeiçoamento desenvolvidos por redes e equipes com um propósito inclusivo".

No Quadro 4, será apresentado o número de publicações por periódicos (sobre os assuntos consultados, conforme resultado da busca).

Quadro 4 - Periódicos com maior ocorrência de publicações sobre a temática abordada

\begin{tabular}{|c|c|c|}
\hline $\mathbf{N}^{\mathbf{0}}$ & Periódico & Frequência \\
\hline 1 & European Journal of Special Needs Education & 4 \\
\hline 2 & International Journal of Inclusive Education & 4 \\
\hline 3 & Revista de Educacion & 4 \\
\hline 4 & Computers Helping People with Special Needs, Icchp 2016, Pt I & 2 \\
\hline 5 & Edulearn15: 7th International Conference on Education and New Learning Technologies & 2 \\
\hline 6 & Inted2014: 8th International Technology, Education and Development Conference & 2 \\
\hline 7 & International Journal of Learning & 2 \\
\hline 8 & Prisma Social & 2 \\
\hline 9 & Revista Brasileira de Educação Especial & 2 \\
\hline 10 & Actual Problems of Economics & 1 \\
\hline 11 & Agro Food Industry Hi-Tech & 1 \\
\hline 12 & Australasian Journal of Special Education & 1 \\
\hline 13 & Center for Educational Policy Studies Journal & 1 \\
\hline 14 & Contextos Educativos-Revista de Educacion & 1 \\
\hline 15 & Critical Studies in Education & 1 \\
\hline 16 & Cyprus International Conference on Educational Research & 1 \\
\hline 17 & Design Education: Collaboration and Cross-Disciplinary & 1 \\
\hline 18 & Education Policy Analysis Archives & 1 \\
\hline 19 & Edulearn11: 3rd International Conference on Education and New Learning Technologies & 1 \\
\hline 20 & Edulearn14: 3rd International Conference on Education and New Learning Technologies & 1 \\
\hline 21 & Elementary School Journal & 1 \\
\hline 22 & Intangible Capital & 1 \\
\hline 23 & International Conference on Educational Robotics, EDUROBOTICS 2016 & 1 \\
\hline 24 & International Conference on New Horizons in Education, Inte 2014 & 1 \\
\hline 25 & International Education Studies & 1 \\
\hline 26 & International Journal of Disability, Development and Education & 1 \\
\hline 27 & International Journal of Economics and Financial Issues & 1 \\
\hline 29 & International Journal of Emerging Technologies in Learning & 1 \\
\hline 30 & International Journal of Special Education & 1 \\
\hline 31 & Journal of Policy and Practice in Intellectual Disabilities & 1 \\
\hline 32 & Journal of Research in Special Educational Needs & 1 \\
\hline 33 & Journal of the Association for Persons with Severe Handicaps & 1 \\
\hline 34 & Novosibirsk State Pedagogical University Bulletin & 1 \\
\hline 35 & Prospects & 1 \\
\hline 36 & Revista Complutense de Educacion & 1 \\
\hline 37 & Revista Electronica de Investigacion Educativa & 1 \\
\hline 38 & Revista Electronica Interuniversitaria de Formacion del Profesorado & 1 \\
\hline 39 & Revista Espanola de Orientacion y Psicopedagogia & 1 \\
\hline 40 & Revista Interuniversitaria de Formacion del Profesorado-Rifop & 1 \\
\hline 41 & Sociologia, Problemas e Práticas & 1 \\
\hline 42 & Support for Learning & 1 \\
\hline 43 & $\begin{array}{l}\text { Third International Conference on Technological Ecosystems for Enhancing Multiculturality, } \\
\text { Proceedings Teem' } 15\end{array}$ & 1 \\
\hline
\end{tabular}

Fonte: Dados da Pesquisa. 
Verificou-se que os periódicos com mais documentos publicados são: "European Journal of Special Needs Education”, “International Journalof Inclusive Education” e "Revista de Educacion", todos com 4 documentos.

A partir do levantamento das palavras-chave encontradas nos trabalhos da busca, foi possível criar uma nuvem de palavras conforme o número de realce das mesmas. Visto que as palavras-chave determinadas para efetuar a busca foram "inclusive education" e innovation, estas não foram consideradas para a construção da nuvem de palavras, uma vez que já representavam os temas da busca, "a ideia é compreender as palavras com maior ocorrência que aparecem associadas à temática do trabalho" (Figura 2).

Figura 2 - Nuvem de palavras-chave em inovação inclusiva na educação

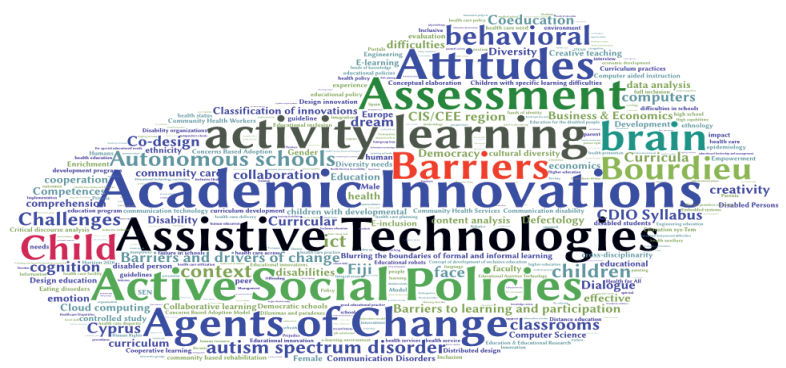

Fonte: Os autores
Conforme a nuvem de palavras, a palavra-chave com maior ocorrência é "Academic Innovations", com maior ocorrência nos trabalhos encontrados na busca, de um total de 400 palavras. Destacam-se também as palavras "Assistive Technologies", "Active Social Policies", com as quais também aparece associado e possuem total relação com a temática, além das palavras "Barriers", "Bourdieu", "Child", entre outras.

O Quadro 5 apresenta a filiação dos autores e países de publicação dos trabalhos mais citados, sobre o tema inovação inclusiva na educação, entre os 19 trabalhos identificados na terceira busca.

Conforme Quadro 5 se verificou também os países de publicação dos 58 documentos identificados para análise (Quadro 6\0.

Pode-se observar que a grande maioria dos documentos identificados, 34\% (20 documentos) foram publicados na Espanha. O restante dos documentos possui origem em vários países.

Quadro 5 - Filiação dos autores e países de publicação dos trabalhos

\begin{tabular}{|c|c|c|c|c|c|}
\hline $\mathbf{N}^{\mathbf{o}}$ & Ano & Autor & Título & Filiação Autor(es) & $\begin{array}{c}\text { País de } \\
\text { Publicação }\end{array}$ \\
\hline 1 & 2016 & $\begin{array}{l}\text { Adayeva e } \\
\text { Satkaliyeva }\end{array}$ & $\begin{array}{l}\text { The innovative management in the field of inclusive } \\
\text { education and its impact on the public health in the } \\
\text { Republic of Kazakhstan }\end{array}$ & Narxoz University & Cazaquistão \\
\hline 2 & 2013 & Alonso, R. F. & $\begin{array}{l}\text { Miguel Catalan secondary school: the strength of } \\
\text { normalcy }\end{array}$ & $\begin{array}{l}\text { Miguel Catalan Secondary } \\
\text { School }\end{array}$ & Espanha \\
\hline 3 & 2015 & Alves et al. & $\begin{array}{l}\text { Exploring technological innovation towards inclusive } \\
\text { education: building digital games - an interdisciplinary } \\
\text { challenge }\end{array}$ & $\begin{array}{l}\text { Universidade do Vale do } \\
\text { Itajaí, Instituto Federal } \\
\text { Catarinense }\end{array}$ & França \\
\hline 4 & 2016 & $\begin{array}{l}\text { Barba-Martin e } \\
\text { Barba }\end{array}$ & $\begin{array}{l}\text { An ongoing collaborative teacher training through } \\
\text { action research. A way of changing classroom } \\
\text { practices }\end{array}$ & Universidade de Valladolid & Espanha \\
\hline 5 & 2013 & Bardy et al. & $\begin{array}{l}\text { Objects for learning as educational resources in } \\
\text { inclusive contexts: support for distance teacher } \\
\text { education }\end{array}$ & $\begin{array}{l}\text { Universidade Federal de São } \\
\text { Carlos }\end{array}$ & Brasil \\
\hline 6 & 2014 & Bari et al. & $\begin{array}{l}\text { The readiness of special education trainees to teach in } \\
\text { inclusive program }\end{array}$ & $\begin{array}{l}\text { Faculty of Education, } \\
\text { University Kebangsaan }\end{array}$ & Malásia \\
\hline 7 & 2013 & $\begin{array}{l}\text { Batanero, J. } \\
\text { M. F. }\end{array}$ & Teaching competences and inclusive education & Universidade de Sevilla & Espanha \\
\hline 8 & 2015 & Benigno et al. & $\begin{array}{l}\text { A web-based knowledge hub for special and inclusive } \\
\text { education }\end{array}$ & University of Salerno & Itália \\
\hline 9 & 2016 & Bernal e Arteaga & $\begin{array}{l}\text { The inquiry of pedagogy in the context of the } \\
\text { inclusive education sociocritical movements }\end{array}$ & $\begin{array}{l}\text { Univ Católica Manizales, } \\
\text { Fac Educ, Manizales }\end{array}$ & Colômbia \\
\hline 10 & 2014 & Blasco et al. & ICT as a model of sensory training in physiotherapy & & Espanha \\
\hline 11 & 2016 & Bosse, I. K. & $\begin{array}{l}\text { Cloud computing in european schools - the impact on } \\
\text { inclusive education }\end{array}$ & Dortmund University & Alemanha \\
\hline 12 & 2010 & Capucha, L. & $\begin{array}{l}\text { Innovation and social justice: active policies for } \\
\text { inclusion in education }\end{array}$ & ISCTE-IUL & Portugal \\
\hline 13 & 2012 & Cortes, R. J. & $\begin{array}{l}\text { Inclusive education and initial teacher training: } \\
\text { evaluation of a didactic innovation based on } \\
\text { filmmaking from a student's perspective }\end{array}$ & Universidade de Málaga & Espanha \\
\hline
\end{tabular}




\begin{tabular}{|c|c|c|c|c|c|}
\hline 14 & 2014 & Deluca et al. & $\begin{array}{l}\text { Promoting the provision of inclusive primary } \\
\text { education for children with disabilities in } \\
\text { mashonaland west province, Zimbabwe }\end{array}$ & UCL & Inglaterra \\
\hline 15 & 2015 & Djukic et al. & $\begin{array}{l}\text { Inclusive education system in Serbia between theory } \\
\text { and practice }\end{array}$ & Univ Novi Sad & Sérvia \\
\hline 16 & 2009 & Echeita et al. & $\begin{array}{l}\text { Paradoxes and dilemmas in the inclusive education } \\
\text { process in Spain }\end{array}$ & & Espanha \\
\hline 17 & 2011 & $\begin{array}{l}\text { Fernandez, M. } \\
\text { S. J. }\end{array}$ & $\begin{array}{l}\text { The innovative model of diversity and high } \\
\text { capabilities attention. "agora model" }\end{array}$ & $\begin{array}{l}\text { Universidad Camilo José } \\
\text { Cela }\end{array}$ & Espanha \\
\hline 18 & 2014 & $\begin{array}{l}\text { Fernandez- } \\
\text { Batanero, J. M. }\end{array}$ & $\begin{array}{l}\text { Strategies for inclusion in the face of social exclusion. } \\
\text { Case study in Andalusia (Spain) }\end{array}$ & Univ Seville & Espanha \\
\hline 19 & 2015 & $\begin{array}{l}\text { Fernandez- } \\
\text { Batanero e Ruiz }\end{array}$ & $\begin{array}{l}\text { Teacher attitudes towards ICT in the context of } \\
\text { inclusive education }\end{array}$ & $\begin{array}{l}\text { University of Sevilla, } \\
\text { University of Jaén }\end{array}$ & Espanha \\
\hline 20 & 2000 & Fisher et al. & $\begin{array}{l}\text { The resilience of changes promoting inclusiveness in } \\
\text { an urban elementary school }\end{array}$ & $\begin{array}{l}\text { The University of Chicago } \\
\text { Press }\end{array}$ & EUA \\
\hline 21 & 2011 & $\begin{array}{l}\text { Florian e } \\
\text { Becirevic }\end{array}$ & $\begin{array}{l}\text { Challenges for teachers' professional learning for } \\
\text { inclusive education in central and eastern Europe and } \\
\text { the commonwealth of independent states }\end{array}$ & $\begin{array}{l}\text { University of Aberdeen, } \\
\text { Open University }\end{array}$ & Reino Unido \\
\hline 22 & 2015 & Gavira e Morina & $\begin{array}{l}\text { Hidden voices in higher education: inclusive policies } \\
\text { and practices in social science and law classrooms }\end{array}$ & Univ Seville & Espanha \\
\hline 23 & 2004 & Ghesquière et al. & $\begin{array}{l}\text { The usefulness of qualitative case studies in research } \\
\text { on special needs education }\end{array}$ & University of Leuven & Bélgica \\
\hline 24 & 2016 & $\begin{array}{l}\text { González Cortés } \\
\text { et al. }\end{array}$ & $\begin{array}{l}\text { Invisible barriers? College student's attitudes towards } \\
\text { peers with disabilities }\end{array}$ & Universidade de Málaga & Espanha \\
\hline 25 & 2014 & Hostins e Jordão & $\begin{array}{l}\text { School inclusion policy and curricular practices: } \\
\text { teaching strategies for target audience conceptual } \\
\text { elaboration of special education }\end{array}$ & $\begin{array}{l}\text { Universidade do Vale do } \\
\text { Itajaí }\end{array}$ & Brasil \\
\hline 26 & 2016 & Infantes et al. & Inclusion as an element of empowerment & & Espanha \\
\hline 27 & 2015 & Kavkler et al. & $\begin{array}{l}\text { Inclusive education for children with specific learning } \\
\text { difficulties: Analysis of opportunities and barriers in } \\
\text { inclusive education in Slovenia }\end{array}$ & University of Ljubljana & Eslovênia \\
\hline 28 & 2010 & $\begin{array}{l}\text { Krasnomovets, } \\
\text { V. A. }\end{array}$ & $\begin{array}{l}\text { Introducing innovations in education as a precondition } \\
\text { for social development of disabled people in Ukraine }\end{array}$ & & Ucrânia \\
\hline 29 & 2017 & Liu et al. & $\begin{array}{l}\text { Research on the computer digital education of special } \\
\text { children based on the theory of multiple intelligences }\end{array}$ & $\begin{array}{l}\text { China Qinhuangdao Haigang } \\
\text { District Xinsheng Road } \\
\text { Primary School }\end{array}$ & China \\
\hline 30 & 2016 & Magnusson, G. & $\begin{array}{l}\text { Representations of special support. Independent } \\
\text { schools' descriptions of special educational provision }\end{array}$ & Mälardalen University & Suécia \\
\hline 31 & 2012 & Marin, S. M. & $\begin{array}{l}\text { Change and innovation in the educational policies and } \\
\text { strategies for human resources development }\end{array}$ & Romanian Academy & Holanda \\
\hline 32 & 2015 & Mesa e Garcia & $\begin{array}{l}\text { Adaptation of Index for Inclusion to the field of higher } \\
\text { education: Preliminary study }\end{array}$ & Universidade de Cantabria & Espanha \\
\hline 33 & 2011 & Moliner et al. & $\begin{array}{l}\text { Inclusive cultures, policies and practices in Spanish } \\
\text { compulsory secondary education schools: teachers' } \\
\text { perceptions in ordinary and specific teaching contexts }\end{array}$ & Universidade de Jaume & Espanha \\
\hline 34 & 2017 & $\begin{array}{l}\text { Montero e } \\
\text { Jormanainen }\end{array}$ & $\begin{array}{l}\text { Theater meets robot - toward inclusive STEAM } \\
\text { education }\end{array}$ & $\begin{array}{l}\text { University of Eastern } \\
\text { Finland }\end{array}$ & Finlândia \\
\hline 35 & 2006 & Mundia, L. & $\begin{array}{l}\text { Aggressive behaviour among Swazi upper primary } \\
\text { and junior secondary students: Implications for } \\
\text { ongoing educational reforms concerning inclusive } \\
\text { education }\end{array}$ & $\begin{array}{l}\text { University of Brunei } \\
\text { Darussalam }\end{array}$ & Brunei \\
\hline 36 & 2006 & Nicolaidou et al. & $\begin{array}{l}\text { Promoting inclusive practices in primary schools } \\
\text { in Cyprus: Empowering pupils to build supportive } \\
\text { networks }\end{array}$ & University of Cyprus & Chipre \\
\hline 37 & 2009 & Ntombela, S. & $\begin{array}{l}\text { Are we there yet? Towards the development of } \\
\text { inclusive education in one district in Kwazulu-Natal, } \\
\text { South Africa }\end{array}$ & $\begin{array}{l}\text { University of Kwa Zulu- } \\
\text { Natal }\end{array}$ & África do Sul \\
\hline 38 & 2016 & Olmos, E. F. T. & $\begin{array}{l}\text { Designing enriched learning environments: a cross- } \\
\text { disciplinary approach to social innovation }\end{array}$ & Aalborg Univ & Dinamarca \\
\hline 39 & 2014 & $\begin{array}{l}\text { Omolade e } \\
\text { Solomon }\end{array}$ & $\begin{array}{l}\text { Perception and use of computer and mobile phones } \\
\text { technologies among visually impaired residents of } \\
\text { Lagos state, Nigeria }\end{array}$ & Lagos State Univ & Nigéria \\
\hline 40 & 1999 & Parrilla, A. & $\begin{array}{l}\text { Educational innovations as a school answer to } \\
\text { diversity }\end{array}$ & $\begin{array}{l}\text { Facultad de Ciencias de la } \\
\text { Educación }\end{array}$ & Espanha \\
\hline 41 & 2017 & Parrilla et al. & $\begin{array}{l}\text { Critically reviewing how we do research into inclusive } \\
\text { education: four projects with an educational and social } \\
\text { approach }\end{array}$ & $\begin{array}{l}\text { Facultad de Ciencias de la } \\
\text { Educación }\end{array}$ & Espanha \\
\hline
\end{tabular}




\begin{tabular}{|c|c|c|c|c|c|}
\hline 42 & 2012 & Pernía et al. & $\begin{array}{l}\text { Student voice in school improvement: Children as } \\
\text { researchers in primary school }\end{array}$ & Univ Cantabria & Espanha \\
\hline 43 & 2016 & Pernía et al. & $\begin{array}{l}\text { Growing student voice in curriculum decisions at the } \\
\text { university }\end{array}$ & Univ Cantabria & Espanha \\
\hline 44 & 2014 & Popescu et al. & $\begin{array}{l}\text { Eyeschool: an educational assistive technology for } \\
\text { people with disabilities - passing from single actors to } \\
\text { multiple-actor environment }\end{array}$ & Univ Paul Sabatier & França \\
\hline 45 & 2009 & Rafal, J. & $\begin{array}{l}\text { Inclusive education: Innovation or merely the } \\
\text { reproduction of hegemonic ideology? }\end{array}$ & University of Cambridge & Reino Unido \\
\hline 46 & 2000 & Rainforth, B. & $\begin{array}{l}\text { Preparing teachers to educate students with severe } \\
\text { disabilities in inclusive settings despite contextual } \\
\text { constraints }\end{array}$ & $\begin{array}{l}\text { University of New York at } \\
\text { Binghamton }\end{array}$ & EUA \\
\hline 47 & 2010 & Reicher, H. & $\begin{array}{l}\text { Building inclusive education on social and emotional } \\
\text { learning: challenges and perspectives - a review }\end{array}$ & Graz Univ & Áustria \\
\hline 48 & 2014 & Reupert et. al. & $\begin{array}{l}\text { Enablers for inclusion: the perspectives of parents of } \\
\text { children with autism spectrum disorder }\end{array}$ & Monash University & Austrália \\
\hline 49 & 2014 & \begin{tabular}{|l|} 
Rosin-Pinola e \\
Del Prette. \\
\end{tabular} & $\begin{array}{l}\text { School inclusion, teacher training and consultation } \\
\text { based on educational social skills }\end{array}$ & $\begin{array}{l}\text { Universidade Federal de São } \\
\text { Carlos }\end{array}$ & Brasil \\
\hline 50 & 2017 & Ryapisova, A. G. & Inclusive education as a systemic innovation & \begin{tabular}{|l|} 
Novosibirsk State \\
Pedagogical University
\end{tabular} & Rússia \\
\hline 51 & 2015 & Sanchez et al. & $\begin{array}{l}\text { Evaluation in primary education as starting point in } \\
\text { the development of inclusive improvement plan in the } \\
\text { Region of Murcia }\end{array}$ & Univ Murcia & Espanha \\
\hline 52 & 2013 & \begin{tabular}{|l|} 
Sánchez-Teruel e \\
Robles-Bello
\end{tabular} & $\begin{array}{l}\text { Inclusion as key to education for all: a theoretical } \\
\text { review }\end{array}$ & Universidad de Jaén & Espanha \\
\hline 53 & 2015 & $\begin{array}{l}\text { Schmidt e } \\
\text { Brown }\end{array}$ & $\begin{array}{l}\text { Education of children with intellectual disabilities in } \\
\text { Slovenia }\end{array}$ & UnivMaribor & Eslovênia \\
\hline 54 & 2015 & Sokal e Katz & $\begin{array}{l}\text { Oh, Canada: bridges and barriers to inclusion in } \\
\text { Canadian schools }\end{array}$ & Univ Winnipeg & Canadá \\
\hline 55 & 2010 & Starcic, A. I. & Educational technology for the inclusive classroom & Univ Primorska & Eslovênia \\
\hline 56 & 2015 & Stoica et al. & Learn the stories from images and beyond & Univ Politehn Bucuresti & Romênia \\
\hline 57 & 2014 & Thomson, $\mathrm{P}$. & $\begin{array}{l}\text { 'Scaling up' educational change: some musings on } \\
\text { misrecognition and doxic challenges }\end{array}$ & Univ Nottingham & Inglaterra \\
\hline 58 & 2003 & Vislie, L. & $\begin{array}{l}\text { From integration to inclusion: focusing global trends } \\
\text { and changes in the western European societies }\end{array}$ & University of Oslo & Noruega \\
\hline
\end{tabular}

Fonte: Dados da pesquisa.

Quadro 6 - Países de publicação dos documentos identificados para análise

\begin{tabular}{|c|c|c|c|c|c|}
\hline País de Publicação & Quantidade & País de Publicação & Quantidade & País de Publicação & Quantidade \\
\hline Espanha & 20 & China & 1 & Ucrânia & 1 \\
\hline Brasil & 3 & Chipre & 1 & & \\
\hline Eslovênia & 3 & Colômbia & 1 & & \\
\hline França & 3 & Dinamarca & 1 & & \\
\hline EUA & 2 & Finlândia & 1 & & \\
\hline Inglaterra & 2 & Holanda & 1 & & \\
\hline Reino Unido & 2 & Itália & 1 & & \\
\hline África do Sul & 1 & Malásia & 1 & & \\
\hline Alemanha & 1 & Nigéria & 1 & & \\
\hline Austrália & 1 & Noruega & 1 & & \\
\hline Áustria & 1 & Portugal & 1 & & \\
\hline Bélgica & 1 & Romênia & 1 & & \\
\hline Brunei & 1 & Rússia & 1 & & \\
\hline Canadá & 1 & Sérvia & 1 & & \\
\hline Cazaquistão & 1 & Suécia & 1 & & \\
\hline
\end{tabular}

Fonte: Dados da Pesquisa.

\section{Conclusão}

Os achados deste estudo fornecem informações aprofundadas sobre o estudo da inovação na educação inclusiva, sua importância, suas barreiras, seus desafios e seu desenvolvimento ao longo do tempo. Ajudam a ilustrar que as inovações impactam toda a sociedade, e ganham um papel fundamental em educação inclusiva, pois norteiam as ações educativas, na promoção de solução para as dificuldades e necessidades apontadas pelo usuário.

O método da pesquisa contribuiu para essa visão, pois a elaboração da folha de cálculos propiciou a percepção de que diversos centros acadêmicos apresentam a preocupação em buscar soluções para os desafios, tanto da inclusão de alunos com deficiências e dificuldades de aprendizagem, quanto para 
os que possuem altas habilidades. Além disso, pode apontar diretrizes promissoras para futuras pesquisas e mais atividades de pesquisa nessa área.

No entanto, a produção científica ainda é recente e requer novos estudos, pois a inovação, além das soluções, contribui para a mudança de paradigma do imobilismo, ainda existente no âmbito educacional. As pesquisas enfatizam a necessidade de mudanças na forma de ensinar, propondo modelos centrados no aluno. Para tal, propõem a formação continuada, cursos e sensibilização do professor e práticas colaborativas de ensino, e-learning, gamificação, o uso da robótica, entre outras.

Enfim, ainda que este estudo não tenha permitido uma cobertura completa de todos os documentos revisados por pares, pode-se afirmar que o processo de revisão abrangeu grande proporção de estudos disponíveis.

\section{Referências}

ADAYEVA, N.; SATKALIYEVA, T. The innovative management in the field of inclusive education and its impact on the public health in the Republic of Kazakhstan. Int. J. Econom. Financial Issues, v.6, n.8, p.370-375, 2016.

ALONSO, R.F. Miguel Catalan Secondary School: the strength of normalcy. Rev. Educ., n.360, p.338-362, 2013.

ALVES, A.G. et al. Exploring technological innovation towards inclusive education: building digital games - an interdisciplinary challenge. In: IAMAN, A.; ESKICUMALI, A. International Conference on New Horizons in Education, Inte 2014. Amsterdam: Elsevier Science, 2015. p.3081-3086.

BARBA-MARTIN, R.A.; BARBA, J.J. An ongoing collaborative teacher training through action research. a way of changing classroom practices. Contextos Educ. Rev Educ., n.19, p.161175, 2016.

BARDIN, L. Análise de conteúdo. Lisbon, 2011.

BARDY, L.R. et al. Objects for learning as educational resources in inclusive contexts: Support for distance teacher education. Rev Bras. Educ. Esp., v.19, n.2, p.273-288, 2013.

BARI, S.; YASIN, M.H.M.; HAMZAH, M.N. The readiness of special education trainees to teach in inclusive program. Int. Educ. Studies, v.7, n.13, p.206-211, 2014.

BATANERO, J.M.F. Teaching competences and inclusive education. Rev Electr. Investig. Educ., v.15, n.2, p.82-99, 2013.

BENIGNO, V.; CAPUANO, N.; MANGIONE, G.R. A webbased knowledge hub for special and inclusive education. Int. J. Emerg. Technol. Learning, v.10, n.7, p.5-13, 2015.

BERNAL, J.C.P.; ARTEAGA, G.A. The Inquiry of pedagogy in the context of the inclusive education Sociocritical movements. Prisma Social, n.16, p.754-770, 2016.

BLASCO, M.C.; BLASCO, J.M.; CASANA, J. ICT As a model of sensory training in physiotherapy. In: CHOVA, L.G. et al. International Technology, Education and Development Conference. Valenica: Iated-Int Assoc Technology Education \& Development, 2014. p.4234-4236.

BOSSE, I.K. Cloud Computing in European Schools - the impact on inclusive education. In: MIESENBERGER, K. et al. Computers helping people with special needs. Cham: Springer Int Publishing, 2016. p.117-120.

CAPUCHA, L. Innovation and social justice: active policies for inclusion in education. Soc. Prob. Prat., v.63, p.25-50, 2010.
CORTES, R.J. Inclusive education and initial teacher training: evaluation of a didactic innovation based on filmmaking from a student's perspective. Rev Educ., n.359, p.232-259, 2012.

DEluCA, M.; TRAMOnTANO, C.; KeTt, M. Promoting The provision of inclusive primary education for children with disabilities in mashonaland west province, zimbabwe. In: CHOVA, L.G. et al.. Inted2014: 8th International Technology, Education and Development Conference. Valenica: IatedIntAssoc Technology Education a\& Development, 2014. p.18161824. (INTED Proceedings).

DJUKIC, M.; DJERMANOV, J.; KOSANOVIC, M. Inclusive education system in serbia between theory and practice. In: GOMEZCHOVA, L. et al. Edulearn 15: 7th International Conference on Education and New Learning Technologies. Valenica: Iated-IntAssoc Technology Education a\& Development, 2015. p.3683-3691.

ECHEITA, G. et al. Paradoxes and dilemmas in the inclusive education process in Spain. Rev Educ, n.349, p.153-178, 2009.

FERENHOF, H.A.; FERNANDES, R.F. Desmistificando a revisão de literatura como base para redação científica: método SFF. Rev ACB, v.21, n.3, p.550-563, 2016.

FERNANDEZ, M.S.J. The innovative model of diversity and high capabilities attention. "Agora ModeL". In: CHOVA, L.G. et al. Edulearn11: 3rd International Conference on Education and New Learning Technologies. Valenica: Iated-IntAssoc Technology Education a\& Development, 2011. p.2046-2049..

FERNANDEZ-BATANERO, J.M. Strategies for inclusion in the face of social exclusion. Case study in Andalusia (Spain). Euro. J. Spec. Needs Educ., v.29, n.3, p.415-428, 2014.

FERNANDEZ-BATANERO, J.M.; RUIZ, M.J.C. Teacher attitudes towards ICT in the Context of Inclusive Education. Third International Conference on Technological Ecosystems for Enhancing Multiculturality, Proceedings Teem'15, p. 111-115, 2015.

FINO, C.M.N. Investigação e inovação (em educação). Pesq. Muda, p.29-48, 2011.

FISHER, D.; SAX, C.; GROVE, K. A. The resilience of changes promoting inclusiveness in an urban elementary school. Elementary School J. v.100, n.3, p.213-227, 2000.

FLORIAN, L.; BECIREVIC, M. Challenges for teachers' professional learning for inclusive education in Central and Eastern Europe and the Commonwealth of Independent States. Prospects, v.41, n.3, p.371-384, 2011.

FREEMAN, A. et al. NMC/CoSN Horizon Report: 2017 K-12 Edition. Austin: The New Media Consortium, 2017

GAVIRA, R.L.; MORINA, A. Hidden voices in higher education: inclusive policies and practices in social science and law classrooms. Int. J. Inclusive Educ., v.19, n.4, p.365-378, 2015.

GHESQUIÈRE, P.; MAES, B.; VANDENBERGHE, R. The usefulness of qualitative case studies in research on special needs education. Int. J. Disab., Develop. Educ., v.51, n.2, p.171-184, 2004.

GONZÁLEZ CORTÉS, E.; ROSES CAMPOS, S. Invisible barriers? College student's attitudes towards peers with disabilities. Rev Compl. Educ., v.27, n.1, p.219-235, 2016.

HOSTINS, R.C.L.; JORDÃO, S.G.F. School inclusion policy and curricular practices: Teaching strategies for target audience conceptual elaboration of special education. Educ. Policy Anal. Arch., v.23, 2014.

INFANTES, A.T.; COLODRERO, V.S.; GUIRAO, J.E.M. Inclusion as an element of empowerment. Prisma Soc., n.16, 
p.348-410, 2016.

JESSON, J.K., MATHESON, L.; LACEY, F.M.. Doing your literature review: traditional and systematic techniques. Sage, Los Angeles, 2011.

KAVKLER, M.; BABUDER, M. K.; MAGAJNA, L. Inclusive education for children with specific learning difficulties: analysis of opportunities and barriers in inclusive education in Slovenia. Center Educ. Policy Studies J., v.5, n.1, p.31-52, 2015.

KRASNOMOVETS, V.A. Introducing innovations in education as a precondition for social development of disabled people in ukraine. Actual Prob. Economics, n. 108, p.199-203, 2010.

LIU, J.; XUE, B.; LIU, J. Research on the computer digital education of special children based on the theory of multiple intelligences. Agro Food Ind. Hi-Tech, v.28, n.1, p.716-720, 2017.

MADARIAGA, A.; RUBIO, I. Videojuegos y discapacidad. El reto de lainclusión. Madrid: IMSERSO, 2012.

MAGNUSSON, G. Representations of special support. Independent schools' descriptions of special educational provision. Euro. J. Spec. Needs Educ., v.31, n.2, p.155-170, 2016.

MARIN, S.M. Change and innovation in the educational policies and strategies for human resources development. In: UZUNBOYLU, H. (Ed.). Cyprus International Conference on Educational Research. Amsterdam: Elsevier Science 2012. p. 1662-1667.

MESA, M.S.; GARCIA, A.I. Adaptation of Index for Inclusion to the field of higher education: Preliminary study. Intangible Capital, v.11, n.3, p.508-545, 2015.

MOLINER, O. et al. Inclusive cultures, policies and practices in Spanish compulsory secondary education schools: teachers' perceptions in ordinary and specific teaching contexts. Int. J. Inclusive Educ., v.15, n.5, p.557-572, 2011.

MONTERO, C.S.; JORMANAINEN, I. Theater meets robot toward inclusive STEAM education. International Conference on Educational Robotics, EDUROBOTICS 2016. ALIMISIS, D. et al: Springer Verlag. 560: 34-40 p. 2017.

MUNDIA, L. Aggressive behaviour among Swazi upper primary and junior secondary students: Implications for ongoing educational reforms concerning inclusive education. Int. J. Spec. Educ., v. 21, n.3, p.58-67, 2006.

NICOLAIDOU, M.; SOPHOCLEOUS, A.; PHTIAKA, H. Promoting inclusive practices in primary schools in Cyprus: Empowering pupils to build supportive networks. Euro. J. Special Needs Educ., v.21, n.3, p.251-267, 2006.

NTOMBELA, S. Are we there yet? Towards the development of inclusive education in one district in Kwazulu-Natal, South Africa. Int. J. Learning, v.16, n.7, p.113-122, 2009.

OLMOS, E.F.T. Designing enriched learning environments: a cross-disciplinary approach to social innovation. Design Education: Collaboration and Cross-Disciplinary, p. 126-131, 2016.

OMOLADE, S.; SOLOMON, M. Perception and use of computer and mobile phones technologies among visually impaired residents of lagos state, Nigeria. In: CHOVA, L.G. et al. Edulearn14: 6th International Conference on Education and New Learning Technologies. Valenica: Iated-IntAssoc Technology Education \& Development, 2014. p.803-812.

PARRILLA, A. et al. Critically Reviewing how we do research into inclusive education: four projects with an educational and social approach. Rev Int. Form. Prof.-Rifop, n.89, p.145-156, 2017.
PARRILLA, A. Educational innovations as a school answer to diversity. Int. J. Inclusive Educ., v.3, n.2, p.93-110, 1999.

PERNÍA, S.R.; SALMÓN, I.H.; LÁZARO-VISA, S. Student voice in school improvement: Children as researchers in primary school. Rev Educ., n.359, p.81-101, 2012.

PERNIA, S.R.; SALMON, I.H.; RADA, T.S. Growing student voice in curriculum decisions at the university. J. Res. Spec. Educ. Needs, v.16, p.563-567, 2016.

POPESCU, C. et al. Eye school: an educational assistive technology for people with disabilities - passing from single actors to multiple-actor environment. In: MIESENBERGER, $\mathrm{K}$. et al. Computers helping people with special needs. Berlin: Springer-Verlag, 2014. p.210-217.

RAFAL, J. Inclusive education: Innovation or merely the reproduction of hegemonic ideology? Int. J. Learning, v.16, n.8, p.235-246, 2009.

RAINFORTH, B. Preparing teachers to educate students with severe disabilities in inclusive settings despite contextual constraints. J. Assoc Persons Severe Handicaps, v.25, n.2, p.83-91, 2000.

REICHER, H. Building inclusive education on social and emotional learning: challenges and perspectives: a review. Int. J. Inclusive Educ., v. 14, n. 3, p. 213-246, 2010.

REUPERT, A.; DEPPELER, J. M.; SHARMA, U. Enablers for Inclusion: the perspectives of parents of children with autism spectrum disorder. Aust. J.Spec. Educ., v.39, n.1, p.85-96, 2014.

ROSIN-PINOLA, A.R.; DEL PRETTE, Z.A.P. School inclusion, teacher training and consultation based on educational social skills. Rev. Bras. Educ. Esp., v.20, n.3, p.341-356, 2014.

RYAPISOVA, A.G. Inclusive education as a systemic innovation. Novosibirsk State Pedag. Univer. Bull., v. 7, n. 1, p. 7-20, 2017.

SANCHEZ, P.A.; RODRIGUEZ, R.D.; LAVELA, J.M.G. Evaluation in primary education as starting point in the development of inclusive improvement plan in the Region of Murcia. Rev. Electr. Int. Forma. Prof., v.18, n.1, p.103-122, 2015.

SÁNCHEZ-TERUEL, D.; ROBLES-BELLO, M.A. Inclusion as key to education for all: a theoretical review. Rev. Espanola Orient. Psicoped., v. 24, n. 2, p. 24-36, 2013.

SANDOBAL, M. et al. Index for inclusion. Una guía para la evaluación y mejora de la educación inclusiva. Contextos Educ., v. $5,227-238,2002$.

SANTOS, G. C. Análise bibliométrica dos artigos publicados como estudos bibliométricos na história do Congresso Brasileiro de Custos. Pensar Contábil, v.17, n.62, 2015

SCHMIDT, M.; BROWN, I. Education of children with intellectual disabilities in Slovenia. J. Policy Practice Intellectual Disab., v.12, n.2, p.90-99, 2015.

SCHUMPETER, J. A teoria do desenvolvimento econômico. São Paulo: Nova Cultural, 1997.

SOKAL, L.; KATZ, J. Oh, Canada: bridges and barriers to inclusion in Canadian schools. Support Learning, v.30, n.1, p.4254, 2015.

STARCIC, A.I. Educational technology for the inclusive classroom. Turkish Online J. Educ. Technol. v.9, n.3, p.26-37, 2010.

STOICA, D.C. et al. Learn the stories from images and beyond. In: GOMEZCHOVA, L.; LOPEZMARTINEZ, A. et al. Edulearn15: 7th International Conference on Education and New Learning Technologies. Valenica: Iated-IntAssoc Technology Education \& Development, 2015. p. 4142-4145. 
THOMSON, P. 'Scaling up' educational change: some musings on misrecognition and doxic challenges. Critical Studies Educ., v. 55, n. 2 , p. $87-103,2014$.
VISLIE, L. From integration to inclusion: Focusing global trends and changes in the western European societies. Euro. J. Special Needs Educ., v.18, n.1, p.17-35, 2003. 